\title{
Disseminated Mycobacterium avium Complex Infection Causing Multiple Skull Defects in an Immunocompetent Patient: A Case Report
}

\author{
Jun Ho Choi (D), Seong Jin Oh (D), Jae Ha Hwang (D), Kwang Seog Kim (D), Sam Yong Lee (D) \\ Department of Plastic and Reconstructive Surgery, Chonnam National University Medical School, Gwangju, Korea
}

\begin{abstract}
Mycobacterium avium complex (MAC) infection mainly causes pulmonary disease. However, in $20 \%$ to $30 \%$ of cases, it also induces various extrapulmonary diseases. Disseminated MAC infection occasionally occurs in immunocompromised patients but very rarely in immunocompetent patients. An 80-year-old immunocompetent woman presented with multiple chronic wounds on the scalp that had not improved despite prolonged treatment. A scalp abscess caused by disseminated MAC infection 4 years ago had gone through repeated cycles of improvement and aggravation despite continued use of anti-mycobacterial agents and active wound care. Enhanced brain computed tomography and magnetic resonance imaging revealed multiple skull defects and abscesses invading the dura mater. Under general anesthesia, the infected scalp skin and bone were sufficiently removed, and the bone and soft tissue defects were repaired with cranioplasty using a titanium mesh plate and local flap. As exemplified in this case, multiple chronic wounds unresponsive to treatment need to be screened for MAC infection. As chronic MAC infection in the scalp can cause skull destruction and brain infection, it needs to be treated aggressively at an early stage to prevent serious morbidity and mortality. Effective MAC infection management involves adequate medication, regular follow-up imaging, and active surgical procedure.
\end{abstract}

Keywords: Mycobacterium avium complex; Epidural abscess; Immunocompetent

\section{Introduction}

Nontuberculous mycobacteria (NTM) are a type of mycobacteria commonly found in the environment, such as water, soil, and animals, and are not transmitted by human-to-human transmission [1]. Among NTM, the most common bacteria causing diseases in humans are the Mycobacterium avium complex (MAC), which consists of Mycobacterium avium and Mycobacterium intracellulare [2].

MAC infection mainly causes respiratory symptoms, but it also causes non-respiratory symptoms such as musculoskeletal symptoms, lymphadenitis, and disseminated infection [3]. Disseminated MAC infection is typically found in immunocompromised patients, including those with acquired immunodeficiency syndrome (AIDS) or malignancy, and is rarely observed in immunocompetent patients [4]. In disseminated MAC infection, skin and soft tissue infection are very common, accompanied by osteomyelitis and bone destruction. Scalp abscess due to disseminated MAC infection can cause skull destruction and brain infection as it progresses.

Here, we report a case of disseminated MAC infection causing multiple skull defects in an immunocompetent patient. The patient provided informed consent for the publication of her clinical and radiological data. The study was exempted from approval by the Institutional Review Board of Chonnam National University Hospital
Case Report

Received: August 13, 2021

Revised: September 22, 2021

Accepted: September 23, 2021

Corresponding author:

Jae Ha Hwang, M.D., Ph.D.

Department of Plastic and Reconstructive Surgery, Chonnam National University Medical School, 42 Jebong-ro, Dong-gu, Gwangju 61469, Korea

Tel: +82-62-220-6354

Fax: +82-62-220-6357

E-mail: psjhhwang@daum.net

This is an Open Access article distributed under the terms of the Creative Commons Attribution Non-Commercial License (https://creativecommons.org/licenses/by-nc/4.0/) which permits unrestricted non-commercial use, distribution, and reproduction in any medium, provided the original work is properly cited.

(c) 2021 Korean Wound Management Society 
(IRB No. CNUH-EXP-2021-285) and performed in accordance with the principles of the Declaration of Helsinki.

\section{Case}

A 76-year-old woman visited the emergency room 4 years prior because of back pain accompanied by fever. The patient had no disease other than hypertension. An increase in inflammatory markers was identified on blood tests, with the following findings: white blood cell $8,900 / \mu \mathrm{L}$, erythrocyte sedimentation rate $108 \mathrm{~mm} / \mathrm{hr}$, and C-reactive protein $18.38 \mathrm{mg} / \mathrm{dL}$. An enhanced magnetic resonance imaging (MRI) test revealed possible infectious spondylitis and multiple paraspinal abscesses at L4-L5, and a diagnosis of spondylitis was made by bone biopsy. The presence of $M$. avium was confirmed using the tuberculosis/nontuberculous mycobacteria-polymerase chain
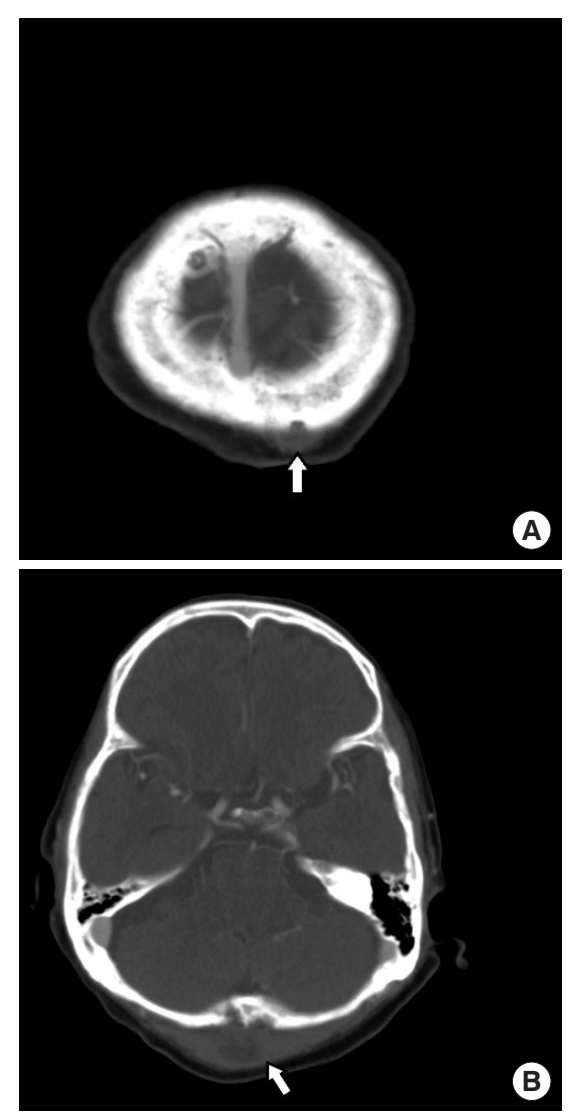

Fig. 1. Imaging study at diagnosis of disseminated Mycobacterium avium complex infection. Multiple abscesses were observed in the scalp on enhanced brain computed tomography. (A) A $2 \times$ $1.5 \times 0.6 \mathrm{~cm}$ fluid collection with thin peripheral enhancement in left parietal scalp, with focal bony erosion (white arrow). (B) A $3.5 \times 3 \times 1 \mathrm{~cm}$ fluid collection with equivocal wall enhancement in occiput area, with underlying bony erosion (white arrow). reaction (TB/NTM-PCR) test. The patient was immunocompetent and started antibiotic treatment at the department of infectious disease, as $M$. avium showed susceptibility to ethambutol, rifampin, amikacin and clarithromycin.

About a week before starting anti-mycobacterial medication, an enhanced brain computed tomography (CT) was performed as fluctuation was palpable in the scalp. Brain CT revealed multiple soft tissue abscesses in the scalp (Fig. 1). Incision and drainage were performed, and MAC was identified in the TB/NTM-PCR test. Later, serial debridement and frequent dressings were performed for the multiple scalp wounds, and secondary intention healing seemed to progress smoothly. The patient had been treated with anti-mycobacterial agents for 3 years. Though a three-drug regimen of ethambutol, rifampin, and clarithromycin was used as the initial therapy, with the emergence of ethambutol-induced optic neuropathy, it was replaced with a three-drug regimen of rifampin, clarithromycin, and amikacin, and the treatment was performed for 3 years and 9 months. However, the wounds repeatedly improved and then deteriorated. Subsequently, the patient was taken off the anti-mycobacterial agents at the department of infectious disease and had received only dressings for a year.

Because of pus discharge from the multiple chronic wounds on the scalp, the patient visited the department of plastic and reconstructive surgery for additional tests (Fig. 2). On enhanced brain CT and MRI, abscesses near the dura mater and chronic osteomyelitis around the skull defect were observed in

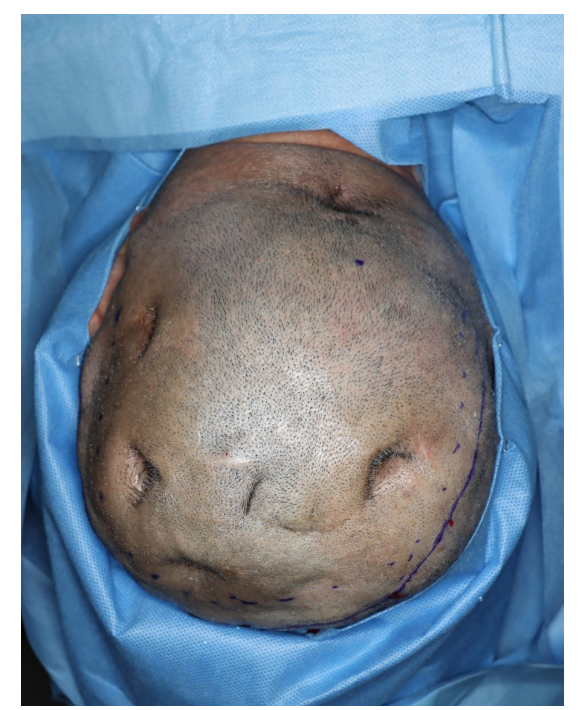

Fig. 2. Preoperative photograph. Multiple soft tissue defects, accompanied by pus-like discharge and bone exposure, were observed on the scalp. 
Choi JH et al.

MAC infection in immunocompetent patient
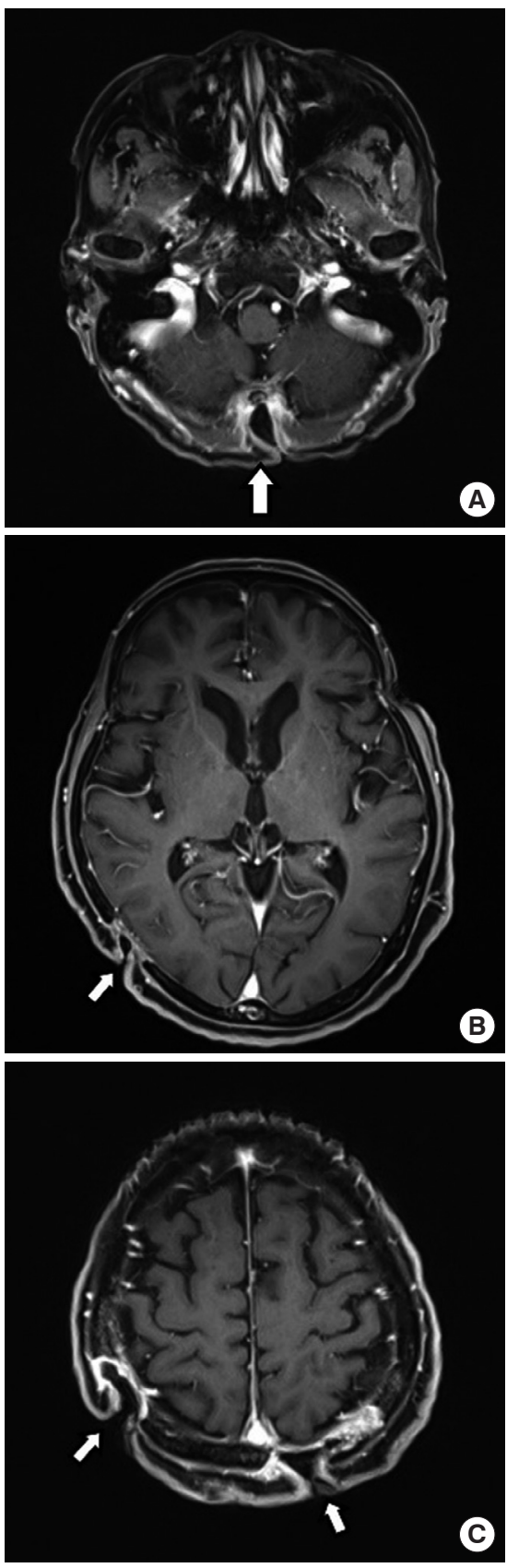

Fig. 3. Preoperative imaging study. Advanced skull destruction and multiple abscesses near the dura mater (white arrows) were observed on the preoperative enhanced brain magnetic resonance imaging.

the right parietal and occipital bones (Fig. 3). Parts of the infected skull and dura mater were subsequently removed under general anesthesia at the department of neurosurgery. Afterwards, cranioplasty was performed using a titanium mesh plate to repair the bone defect. Finally, the scalp skin and soft tissue were sufficiently removed and the soft tissue defects
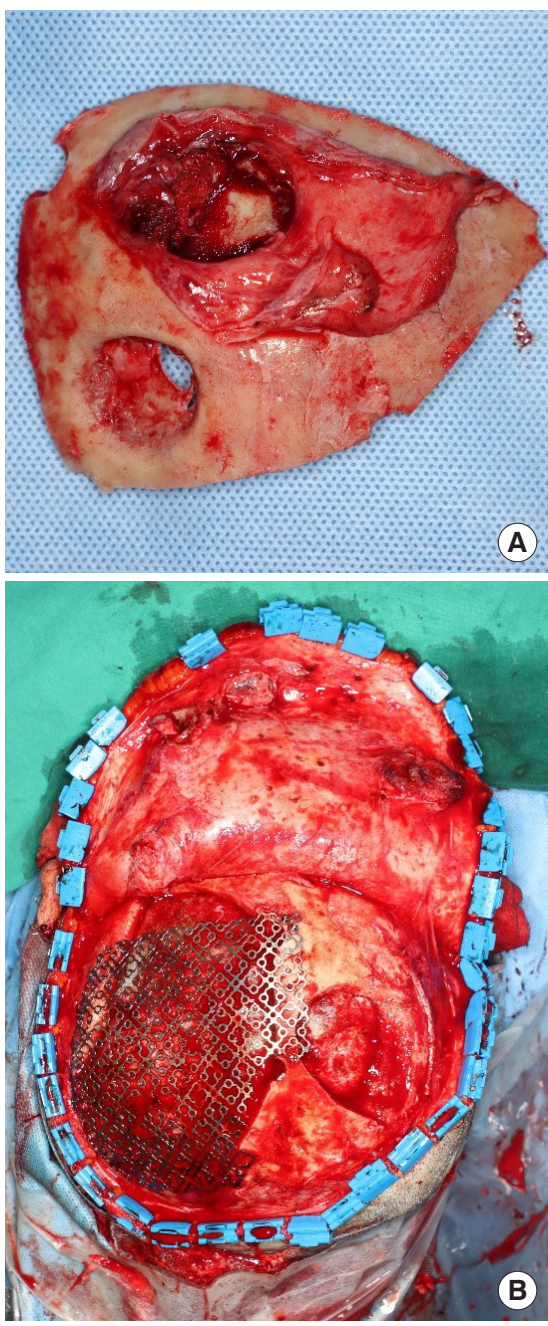

Fig. 4. Intraoperative photographs. (A) Removed parietal bone. Concomitant bony defect and osteomyelitis were observed. (B) Intraoperative photograph obtained after fixation with a mesh plate following removal of the involved bone.

were repaired with a local advancement flap at the department of plastic and reconstructive surgery (Fig. 4). After surgery, on consulting with the department of infectious disease, the patient was not treated with anti-mycobacterial agents and only used prophylactic antibiotics for surgery. The patient recovered well without any complications. During the 6-month follow-up period, there was no wound dehiscence or recurrence of abscess (Fig. 5).

\section{Discussion}

NTM are commonly found in environments inhabited by humans. They have a low virulence but occasionally cause infections in immunocompromised patients, such as those with 


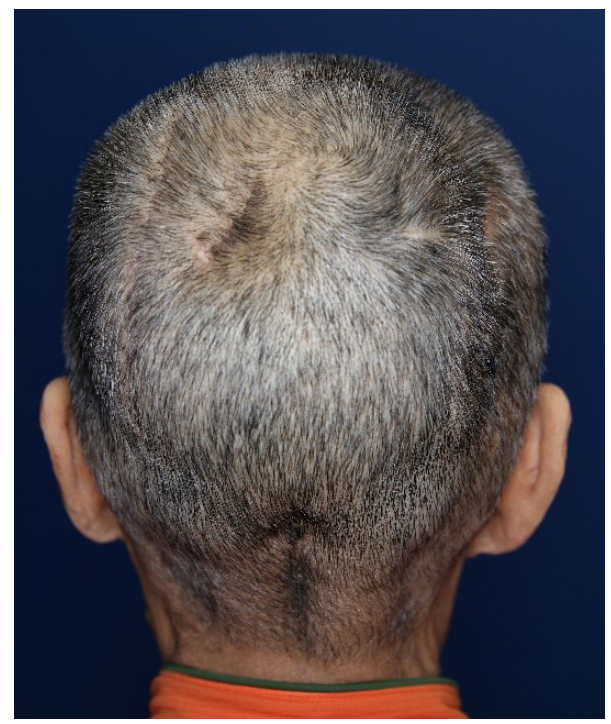

Fig. 5. Postoperative photograph. Wounds have recovered well without complications such as wound dehiscence or abscess recurrence.

AIDS. Diseases due to NTM infection can be largely classified into pulmonary and extrapulmonary diseases, including skin and soft tissue infection, musculoskeletal infection, lymphadenitis, and disseminated disease [5]. Most NTM infections appear in the form of pulmonary diseases, and $20 \%$ to $30 \%$ of cases appear in the form of extrapulmonary diseases [3]. Mycobacterium intracellulare is the most common causative bacteria for pulmonary diseases, accounting for $38.9 \%$ of infections; and M. avium is the most common causative bacteria for extrapulmonary diseases, accounting for $25.0 \%$ of infections. These two bacterial species are classified as MAC, being the most common bacteria accounting for approximately $61 \%$ of NTM infections in humans [2]. In addition, the MAC includes the most common NTM causing disseminated disease, and therefore precise knowledge and treatment are required [5]. Disseminated MAC infection mainly occurs in immunocompromised patients and occurs very rarely in immunocompetent patients [4]. The patient in this case was being treated for hypertension, with no disease-causing immunodeficiency or related drugs administered.

The cause of MAC infection in immunocompetent patients remains unclear, although it is assumed that MAC infection is related to variations in the synthesis and action pathways of interferon- $\gamma$ and interleukin-12 [6]. Multiple recent studies have reported an association between disseminated MAC infection in immunocompetent patients and interferon- $\gamma$ autoantibodies [7]. Dissemination of MAC is thought to begin from infection of mucosal surfaces such as the lungs and intestines, followed by local proliferation and invasion into other organs and tissues of the body through the bloodstream [2].

The most common clinical symptoms of disseminated MAC infection include persistent fever, night sweats, weight loss, fatigue, lethargy, and loss of appetite [6]. Other common symptoms include skin and soft tissue infections. Cutaneous infections occur in various forms, including abscesses, nodular lesions, erythematous plaques with yellow crust, ulceration, panniculitis, tenosynovitis, and fasciitis [8]. In this case, the patient had spondylitis, paravertebral abscess, abscess, bone destruction in the femoral neck, and multiple abscesses in the sternum and scalp, with concomitant skull destruction in the scalp abscess. The patient also showed mild adhesion because of the presence of inflammatory tissue up to the surface of the dura mater through skull defects, whereas infection such as abscess was not observed within the central nervous system (CNS). Although CNS infection due to MAC is very rare in immunocompetent patients, it is associated with a high mortality rate of $35 \%-70 \%$ [6]. It therefore should be considered as a possibility while diagnosing patients with disseminated infections. In the case of concomitant infection in the scalp, as in this case, examination of CNS infection and regular follow-up seem necessary, as invasion into the CNS may take place after skull destruction.

A diagnosis of MAC infection needs to be made in cooperation with specialists from various fields. Because diagnoses based on clinical symptoms and diagnostic imaging lack sensitivity and specificity, culture is the gold standard for making a precise diagnosis. PCR tests, including tissue culture, can precisely identify MAC, while drug susceptibility testing is useful for the selection of appropriate medication [5]. Concurrent therapy with surgical debridement and chemotherapy is recommended for the treatment of MAC infections. General MAC infection requires treatment for 6-12 months, with a minimum of three drugs to reduce drug resistance. The macrolide class of antibiotics is important for treatment; triple therapy mainly consists of clarithromycin, ethambutol, and rifampin, with some modifications depending on drug susceptibility of bacteria and the occurrence of side effects [3].

Although MAC infections are considered rare, it is advisable to screen for them in cases where there are multiple chronic wounds that are unresponsive to treatment. In particular, early diagnosis and treatment must be conducted because chronic scalp MAC infection has a higher risk of causing complications, such as bone destruction and CNS infection. For the 
treatment of MAC infection, medication, wound care, regular diagnostic imaging, and active surgical procedures like wide debridement and flap surgery are required.

\section{Conflict of interest}

Jae Ha Hwang is an editorial board member of the journal but was not involved in the peer reviewer selection, evaluation, or decision process of this article. No other potential conflicts of interest relevant to this article were reported.

\section{ORCID iDs}

$\begin{array}{ll}\text { Jun Ho Choi } & \text { https://orcid.org/0000-0002-4848-517X } \\ \text { Seong Jin Oh } & \text { https://orcid.org/0000-0003-4057-9398 } \\ \text { Jae Ha Hwang } & \text { https://orcid.org/0000-0001-6992-8067 } \\ \text { Kwang Seog Kim } & \text { https://orcid.org/0000-0002-6766-4640 } \\ \text { Sam Yong Lee } & \text { https://orcid.org/0000-0002-3185-2519 } \\ \text { References } & \end{array}$

1. Yu K, Song L, Zhang J, et al. A young boy with disseminated Mycobacterium avium complex infection. Int J Infect Dis 2019;81:10-1.
2. Chung JW, Cha YJ, Oh DJ, et al. Disseminated Mycobacterium avium complex infection in a non-HIV-infected patient undergoing continuous ambulatory peritoneal dialysis. Korean J Lab Med 2010;30:166-70.

3. Wi YM. Treatment of extrapulmonary nontuberculous mycobacterial diseases. Infect Chemother 2019;51:245-55.

4. Stewart A. Disseminated Mycobacterium intracellulare from a deep cutaneous infection. Int J Mycobacteriol 2019; 8:98-100.

5. Xu X, Lao X, Zhang C, et al. Chronic Mycobacterium avium skin and soft tissue infection complicated with scalp osteomyelitis possibly secondary to anti-interferon- $\gamma$ autoantibody formation. BMC Infect Dis 2019;19:203.

6. Chowdhary M, Narsinghani U, Kumar RA. Intracranial abscess due to Mycobacterium avium complex in an immunocompetent host: a case report. BMC Infect Dis 2015; $15: 281$.

7. Ikeda $\mathrm{H}$, Nakamura K, Ikenori M, et al. Severe disseminated Mycobacterium avium infection in a patient with a positive serum autoantibody to interferon- $\gamma$. Intern Med 2016; 55:3053-8.

8. Pampaloni A, Tosto S, Locatelli ME, et al. Skin and soft tissue infection by Mycobacterium intracellulare in an immunocompetent patient. IDCases 2020;19:e00720. 